\title{
UMA PROVA TOPOLÓGICA DO TEOREMA DE RAMSEY
}

A TOPOLOGICAL PROOF OF RAMSEY'S THEOREM

MÁRCIO TELLES \#

JEFFERSON WALLACE $\rrbracket$

\begin{abstract}
Este texto apresenta uma demonstração do Teorema de Ramsey usual, usando a estrutura de um espaço topológico ultramétrico compacto, e pode ser usado como uma introdução à Teoria de Ramsey. Também fornece o contexto histórico para algumas das sutilezas de Lógica e Fundamentos envolvidas nos argumentos.
\end{abstract}

Palavras-chave: Combinatória, Matemática Discreta, Topologia, Lógica.

\begin{abstract}
This text presents a proof of Ramsey's Theorem, using the structure of a compact ultrametric space, and can be used as an introduction to Ramsey Theory. Also, the historical context for some of the logical subleties that occur in the arguments is given.
\end{abstract}

Keywords: Combinatorics, Discrete Mathematics, Topology, Logic.

MSC2010: 0501, 5401, 0301

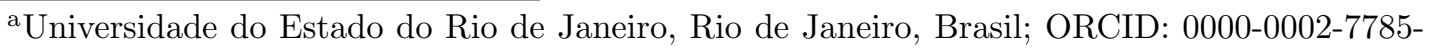
6662 E-mail: marcio.telles@uerj.br

b ORCID: 0000-0001-8067-6139 E-mail: profjeffersonmaciel@gmail.com
} 


\section{Introdução}

A Teoria de Ramsey é um assunto muito vasto e fascinante, cujas origens e motivações remontam a discussões elementares no contexto da Análise Combinatória. Menções explícitas a seus teoremas mais conhecidos começam a aparecer no início do século XX, especialmente nos trabalhos do Matemático Britânico Frank P. Ramsey, que empresta seu nome à teoria. Hoje, esta teoria tem escopo de aplicabilidade e pesquisa muito amplos, chegando, por exemplo, às fronteiras da Combinatória Infinitária.

O presente texto tem dois objetivos principais. O primeiro é servir como um convite introdutório à Teoria de Ramsey, motivando-a através de exemplos simples e guiando a intuição do leitor paulatinamente a dois dos seus resultados seminais, chamados aqui de Versão Finita e Versão Infinita do Teorema de Ramsey. O segundo é apresentar os detalhes de como a compacidade de um certo espaço métrico pode ser usada como ingrediente fundamental na demonstração do conhecido fato de a Versão Infinita implicar a Versão Finita.

O restante das notas estrutura-se da seguinte forma: a seção 2 apresenta motivações para a Teoria de Ramsey, partindo de fatos simples de combinatória básica, e culmina no enunciado da Versão Finita do Teorema de Ramsey. A seção 3 contém o enunciado e a demonstração da Versão Infinita do Teorema de Ramsey. A seção 4 apresenta um espaço métrico compacto, cuja estrutura é claramente relacionada aos conceitos estudados anteriormente. A seção 5 utiliza este conjunto compacto para demonstrar uma variante mais forte da Versão Finita, chamada de Versão de ParisHarrington. A última seção contém elementos históricos que justificam nossa escolha de mencionar a Versão de Paris-Harrington, inserindo-a no contexto da dicotomia entre métodos finitários e infinitários em Matemática: esta dicotomia foi palco de uma conhecida controvérsia no final do século XIX. O Teorema de Paris-Harrington, enunciado também na última seção, em conjunto com uma revisão cuidadosa de todo o texto, ajuda a iluminar alguns aspectos muitos sutis de toda essa discussão.

Apesar de referências à possibilidade do uso das técnicas topológicas que discutimos acima em demonstrações do Teorema Finito de Ramsey existirem em literaturas variadas, não encontramos os argumentos completos escritos em detalhe e, portanto, achamos que incluí-los no presente trabalho seria proveitoso.

Esperamos que este texto dê sua modesta contribuição à divulgação e ao incremento do interesse por esses interessantíssimos temas. 


\section{Ramsey: Versão Finita}

Em um sentido muito preciso, a ser definido mais adiante, a desordem completa não existe segundo a Teoria de Ramsey. Essa teoria investiga a ordem inerente de algumas estruturas matemáticas. Quão grande esta deve ser, de modo a assegurar que pelo menos uma das partes tenha determinada propriedade? O exemplo a seguir é uma instância simples e emblemática desse estado de coisas.

Em uma festa com seis pessoas, podemos afirmar que três delas se conhecem ou três delas se desconhecem.

Esse é um fato bastante conhecido no contexto de Combinatória Elementar. Ao reinterpretarmos cada pessoa como o vértice de um grafo completo, cujas arestas são pintadas de azul ou vermelho de acordo com as pessoas correspondentes se conhecerem ou se desconhecerem, obtemos a seguinte reformulação:

Proposição 1. Se colorirmos, usando duas cores distintas, cada uma das 15 arestas do grafo completo em 6 vértices, haverá pelo menos um triângulo monocromático, isto é, cujas arestas são todas da mesma cor.

Proof. Escolha um vértice $v$ e note que pelo menos 3 arestas incidindo em $v$ são da mesma cor, digamos azul. Sejam tais arestas $e_{1}=\{v, x\}, e_{2}=\{v, y\}$ e $e_{3}=\{v, z\}$. Se o triângulo $\{x, y, z\}$ for monocromático, terminamos. Caso contrário, alguma de suas arestas é azul, digamos (sem perda de generalidade) $\{x, y\}$. Assim, o triângulo $\{v, x, y\}$ é monocromático.

Uma maneira natural de abordar a Teoria de Ramsey é generalizar, passo a passo, alguns parâmetros nessa proposição. Comecemos impondo, em vez de triângulos, objetos monocromáticos formados por um número possivelmente maior de vértices:

Dados $s$ e $t$ inteiros positivos, qual deve ser o número mínimo $n$ de pessoas em uma festa, de modo a garantir que existem $s$ pessoas que se conhecem ou existem $t$ pessoas que não se conhecem? O número de Ramsey, denotado por $R_{2}(s, t)$, é o menor número que satisfaz a condição anterior. A proposição acima significa que $R_{2}(3,3) \leq 6$. Além disso, é fácil colorir as arestas de um grafo completo em 5 vértices de forma a não haver triângulos monocromáticos, o que nos dá a infomação exata $R_{2}(3,3)=6$.

A despeito de sua definição conceitualmente simples, a prática tem mostrado que é muito difícil calcular números de Ramsey explicitamente em geral. Por exemplo, hoje, ainda não se conhece o valor de $R_{2}(5,5)$ ! Mas vale a pena mencionar, de passagem, que $43 \leq R_{2}(5,5) \leq 48$ e conjectura-se que $R_{2}(5,5)=43$ (ver [6]). 
Aliás, é muito importante notar que, a princípio, não é nem claro que tais números estejam bem definidos! O fato de que eles estão é um dos muitos teoremas que levam o nome de Ramsey, e será o foco da nossa atenção neste texto. A fim de enunciá-lo, vamos generalizar não apenas o número de variáveis da função $R_{2}$ como também o subíndice 2 .

Como motivação, mencionamos que, em um conjunto de 17 pessoas, podemos encontrar três pessoas que se amam, ou três pessoas que se odeiam, ou três pessoas que são indiferentes entre si. Além disso, nenhum número menor do que 17 possui essa propriedade. Expressamos sucintamente as informações acima dizendo que o número de Ramsey $R_{2}(3,3,3)$ é igual a 17.

Continuando a subir os degraus de generalidade, passamos a considerar também hipergrafos, em que as "arestas" podem ter mais do que 2 vértices:

Definição 1. Dados e, $a_{1}, a_{2}, \ldots, a_{r}, \in \mathbb{N}$, o Número de Ramsey $R_{e}\left(a_{1}, \ldots, a_{r}\right)$, caso exista, é o menor inteiro $N$ tal que, para todo conjunto $X$ com pelo menos $N$ elementos e toda função $f:\left(\begin{array}{c}X \\ e\end{array}\right) \rightarrow\left\{c_{1}, \ldots, c_{r}\right\}$, existe $1 \leq i \leq r$ e um subconjunto $Y \subseteq X$ com $a_{i}$ elementos tal que $C$ é constante em $\left(\begin{array}{l}Y \\ e\end{array}\right)$.

Repare que, na notação $\left(\begin{array}{l}X \\ e\end{array}\right)$, temos uma importante diferença em relação ao número binomial usual. Aqui, o índice superior $X$ é um conjunto. Nesse caso, $\left(\begin{array}{l}X \\ e\end{array}\right)$ é a classe dos subconjuntos de $X$ de tamanho $e$. Assim, o número de elementos do conjunto $\left(\begin{array}{c}X \\ e\end{array}\right)$ é $\left(\begin{array}{c}|X| \\ e\end{array}\right)$.

Na definição acima, é instrutivo pensar que estamos colorindo as arestas do hipergrafo completo e-regular (i.e., o hipergrafo com vértices em X cujas arestas são todos os elementos de $\left.\left(\begin{array}{c}X \\ e\end{array}\right)\right)$ com as $r$ cores $c_{1}, \ldots, c_{r}$. O conjunto $Y$ mencionado é um subhipergrafo induzido de $X$, monocromático para a coloração $f$.

Como já mencionamos, não é claro que números de Ramsey existam para todas as escolhas dos parâmetros mencionados. Mas, de fato, eles sempre existem:

Teorema 1 (Ramsey, Versão Finita). Dados e, $a_{1}, \ldots, a_{r} \in \mathbb{N}$, existe o número de Ramsey $R_{e}\left(a_{1}, \ldots, a_{r}\right)$.

Uma das demonstrações deste teorema consiste de várias induções aninhadas, acompanhando as generalizações dos parâmetros na ordem que descrevemos acima, tendo como caso-base a proposição 1, com sua demonstração inspirando parte dos argumentos (ver [1] ou [14]). Aqui, vamos seguir um caminho um pouco mais longo. Primeiramente, na próxima seção, apresentaremos um outro teorema, que pode ser interpretado como uma "versão infinita" do Teorema de Ramsey. 


\section{Ramsey: Versão Infinita}

Uma maneira possível de interpretar $a_{1}=a_{2}=\ldots=a_{r}=\infty$ no teorema que examinamos no final da seção anterior é o enunciado abaixo, que, naturalmente, merece a alcunha de "Versão Infinita" do Teorema de Ramsey. A demonstração que apresentamos, devida a Erdös, se faz por indução em $e$. Note como os procedimentos iniciais do passo indutivo estão em clara analogia ao início da demonstração da Proposição 1.

Teorema 2 (Ramsey, Versão Infinita). Seja X um conjunto infinito e pinte os elementos de $\left(\begin{array}{c}X \\ e\end{array}\right)$ com $r$ cores diferentes, segundo a coloração $f:\left(\begin{array}{c}X \\ e\end{array}\right) \rightarrow\left\{c_{1}, \ldots, c_{r}\right\}$. Então existe $Y \subseteq X$ infinito e monocromático, i.e., tal que $f$ é constante em $\left(\begin{array}{l}Y \\ e\end{array}\right)$.

Proof. A prova será por indução em $e$.

Para $e=1$, o enunciado é apenas a afirmação obviamente verdadeira de que, se infinitos objetos são distribuídos em um número finito de caixas, então haverá pelo menos uma caixa contendo infinitos objetos.

Supondo o resultado válido para $e$, devemos mostrar que ele é válido para $e+1$.

Seja $f:\left(\begin{array}{c}X \\ e+1\end{array}\right) \rightarrow\left\{c_{1}, \ldots, c_{r}\right\}$, e fixe $x_{0} \in X$ arbitrário. Vamos construir uma coloração $\tilde{f}$ "induzida" por $f$ em $\left(\begin{array}{c}X \backslash\left\{x_{0}\right\} \\ e\end{array}\right)$, impondo

$$
\tilde{f}\left(y_{1}, y_{2}, \ldots, y_{e}\right)=f\left(y_{1}, y_{2}, \ldots, y_{e}, x_{0}\right)
$$

Pela hipótese de indução aplicada a $\tilde{f}$, existe $X_{1} \subseteq X \backslash\left\{x_{0}\right\}$ infinito monocromático. Esta cor será chamada de cor de referência de $x_{0}$. Note que a presença de $x_{0} \mathrm{em}$ qualquer aresta cujos outros vértices estejam em $X_{1}$ determina a $f$-cor desta aresta: a cor de referência de $x_{0}$.

Repetimos o argumento, agora começando com $X_{1}$, e obtendo $x_{1}$ (com sua cor de referência) e $X_{2}$. Mais geralmente, prosseguimos, supondo definidos $x_{k-1}$ (e sua cor de referência) e $X_{k}$, aplicamos o mesmo argumento ao conjunto $X_{k}$, obtendo $x_{k}$ (e sua cor de referência) e $X_{k+1}$.

Finalmente, novamente usando o fato óbvio citado no caso-base $e=1$, existe um subconjunto infinito $Y \subseteq\left\{x_{0}, x_{1}, x_{2}, \ldots\right\}$ monocromático para a cor de referência (note que o número de cores de referência é $\leq r$ ). É claro que $Y$ é monocromático para $f$.

A versão infinita do Teorema de Ramsey é uma das duas peças fundamentais que usaremos para demonstrar a versão finita. A outra peça é um espaço topológico, 
que introduziremos na seção a seguir (para uma construção relacionada, vide [17]).

\section{O Espaço das Colorações}

Com o objetivo de usar conceitos de topologia para demonstrar o Teorema Finito de Ramsey, vamos construir um espaço métrico $\mathcal{K}$, cujo conjunto de pontos será a classe de todas as colorações dos subconjuntos de $\mathbb{N}$ com $e$ elementos usando as $r$ cores no conjunto $C=\left\{c_{1}, \ldots, c_{r}\right\}$. Mais sucintamente,

$$
\mathcal{K}:=\left\{f \mid f:\left(\begin{array}{c}
\mathbb{N} \\
e
\end{array}\right) \rightarrow C\right\} .
$$

Vamos definir uma métrica $d$ em $\mathcal{K}$, que o tornará um espaço métrico compacto. No que vai a seguir, dado $m \in \mathbb{N},[m]:=\{0,1,2, \ldots, m-1\}$.

Definição 2. Sejam $f, g \in \mathcal{K}$. Se $f=g$, definimos $d(f, g):=0$. Se $f \neq g$, seja $k o$ (único) natural tal que $f \uparrow_{\left(\begin{array}{c}{[k]} \\ e\end{array}\right)}=g \uparrow_{\left(\begin{array}{c}{[k]} \\ e\end{array}\right)}$ mas $f \uparrow_{\left(\begin{array}{c}{[k+1]} \\ e\end{array}\right)} \neq g \uparrow_{\left(\begin{array}{c}{[k+1]} \\ e\end{array}\right)}$. Então, definimos $d(f, g):=2^{-k}$.

Vamos mostrar que $d$ é uma métrica em $\mathcal{K}$.

Teorema 3. $(\mathcal{K}, d)$ é um espaço métrico.

Proof. É fácil ver que $d \geq 0$ e que $d(f, g)=d(g, f)$ quaisquer que sejam $f$ e $g$. Também é imediato ver que $d(f, g)=0 \Longleftrightarrow f=g$.

Resta provar a desigualdade triangular, o que faremos através da desigualdade abaixo, obviamente suficiente, porque mais forte (esta desigualdade faz de $d$ uma ultramétrica):

$$
d(f, g) \leq \max \{d(f, h), d(h, g)\} .
$$

Para provar a desigualdade acima, sejam

$$
\begin{aligned}
d_{1} & :=d(f, g)=2^{-k_{1}} \\
d_{2} & :=d(f, h)=2^{-k_{2}} \\
d_{3} & :=d(h, g)=2^{-k_{3}} \\
k & :=\min \left\{k_{2}, k_{3}\right\}
\end{aligned}
$$

e repare que, como $f\left\lceil_{\left(\begin{array}{c}{[k]} \\ e\end{array}\right)}=g\left\lceil_{\left(\begin{array}{c}{[k]} \\ e\end{array}\right)}\right.\right.$, temos $k_{1} \geq k$ e, portanto

$$
d_{1}=2^{-k_{1}} \leq 2^{-k}=\max \left\{d_{2}, d_{3}\right\}
$$


É conveniente compreender o espaço $\mathcal{K}$ sob uma outra perspectiva: a das árvores, como segue abaixo:

Uma árvore enraizada é um grafo conexo e acíclico em que está destacado um único vértice, chamado de raiz. A raiz constitui o nível 0 da árvore, e seus vizinhos imediatos, o nível 1 . Indutivamente, o vértice $v$ está no nível $k+1$ se, e somente se, não estiver no nível $k-1$ e for vizinho imediato de algum vértice no nível $k$. Se $v$ e $w$ são vizinhos, $v$ está no nível $k$ e $w$ está no nível $k+1$, dizemos que $v$ é pai de $w$ ou que $w$ é filho de $v$.

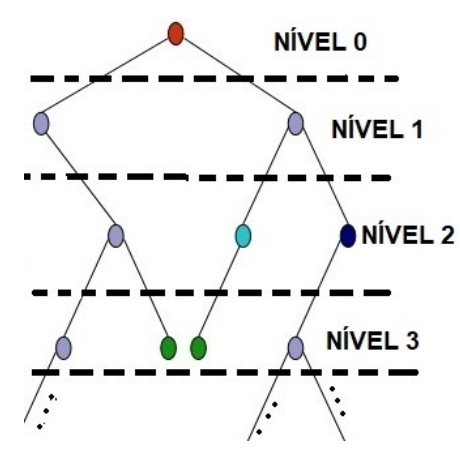

Figure 1: Uma árvore cuja raiz é o vértice vermelho.

Considere qualquer árvore infinita enraizada, em que cada nível é finito. Os pontos do espaço métrico $\mathcal{K}$ que temos em mente são os galhos da árvore, i.e., os caminhos infinitos nos vértices, partindo da raiz, em que cada vértice no caminho é filho de seu antecessor imediato.

Agora, para definirmos a métrica, tome dois pontos

$$
v=\left(a_{0}, a_{1}, \ldots\right), \quad w=\left(b_{0}, b_{1}, \ldots\right) \in \mathcal{K} .
$$

Obviamente, se $v=w$, poremos $d(v, w)=0$. Por outro lado, se $v \neq w$ e $a_{i}=b_{i}$ para todo $0 \leq i \leq k$ mas $a_{k+1} \neq b_{k+1}$, definimos a distância como:

$$
d(v, w)=2^{-k}
$$

Considere, novamente, $\mathcal{K}=\left\{f:\left(\begin{array}{c}\mathbb{N} \\ e\end{array}\right) \rightarrow C\right\}$, o espaço das colorações. Podemos usá-lo para induzir a construção de uma árvore como aquela descrita acima, da seguinte forma:

1) Cada um dos galhos (caminhos infinitos) da árvore é uma coloração $f:\left(\begin{array}{l}\mathbb{N} \\ e\end{array}\right) \rightarrow$ $C$, um elemento de $\mathcal{K}$ (nos dois sentidos!). 
2) Cada vértice da árvore no nível $k$ é a restrição de uma coloração "completa" (com domínio $\left(\begin{array}{c}\mathbb{N} \\ e\end{array}\right)$ ) a uma coloração com domínio $\left(\begin{array}{c}{[k]} \\ e\end{array}\right)$. Dois vértices são vizinhos se as colorações correspondentes são vizinhas, i.e., uma é a restrição da outra.

Em outras palavras, e unificando as duas perspectivas, a coloração $f:\left(\begin{array}{l}\mathbb{N} \\ e\end{array}\right) \rightarrow C$ está identificada com a sequência (galho da árvore) $v=\left(a_{0}, a_{1}, \ldots\right)$, onde, para cada $k$,

$$
a_{k}=f \Upsilon_{\left(\begin{array}{c}
{[k]} \\
e
\end{array}\right)} \cdot
$$

Assim, a métrica da árvore é igual à métrica do espaço $\mathcal{K}=\left\{f:\left(\begin{array}{c}\mathbb{N} \\ e\end{array}\right) \rightarrow C\right\}$, que havíamos apresentado anteriormente.

Já sabemos, então, que $\mathcal{K}$ (nas duas interpretações apresentadas!) é um espaço métrico.

Além disso, segundo uma das versões usuais do Lema de König, qualquer espaço métrico construído a partir de uma estrutura de árvore como acima é compacto:

Teorema 4 (König). O espaço $\mathcal{K}$ é compacto.

Proof. Já sabemos que $\mathcal{K}$ é um espaço métrico. Então, basta provarmos que toda sequência $v^{n}=\left(a_{0}^{n}, a_{1}^{n}, a_{2}^{n}, \ldots\right)$ de elementos de $\mathcal{K}$ possui uma subsequência convergente.

Vamos exibir uma subsequência $v^{n_{j}}$ convergindo a $w=\left(b_{0}, b_{1}, b_{2} \ldots\right)$, construindo esses objetos por indução:

Começamos notando que, necessariamente, $b_{0}$ é a raiz. Poremos, também, $n_{0}=$ 0 , ou seja, $v^{n_{0}}=v^{0}$. Como o nível 1 é finito, há infinitos termos da sequência $v^{n}$ com a mesma segunda coordenada, digamos $b_{1}$. Escolhemos um desses termos $v^{n_{1}}$ de forma que $n_{1}>n_{0}$, o que é possível, pois há infinitos termos da sequência com segunda coordenada igual a $b_{1}$. Desprezamos todos os outros termos da sequência, i.e., todos os termos que não têm segunda coordenada igual a $b_{1}$, e repetimos o argumento com os infinitos termos da sequência cuja primeira coordenada é $b_{0}$ e a segunda coordenada é $b_{1}$ :

Suponha definidos $b_{0}, b_{1}, \ldots, b_{l}$ de maneira que haja infinitos termos da sequência $v^{n}$ da forma $\left(b_{0}, b_{1}, \ldots, b_{l}, \ldots\right)$ e $n_{l}$ tal que os termos $v^{n_{0}}, v^{n_{1}}, \ldots, v^{n_{l}}$ satisfazem $n_{0}<n_{1}<\ldots<n_{l}$ e, para cada $j \in\{1,2, \ldots, l\}, v^{n_{j}}=\left(b_{0}, b_{1}, \ldots, b_{j}, \ldots\right)$. Como o nível $l+1$ da árvore é finito, existem infinitos termos da sequência $v^{n}$ da forma $\left(b_{0}, b_{1}, \ldots, b_{l}, \ldots\right)$ com a mesma $(l+1)$-ésima coordenada, digamos $b_{l+1}$. Escolhemos um desses termos $v^{n_{l+1}}$ de forma que $n_{l}<n_{l+1}$, o que é possível, dada a infinitude das possíveis escolhas. Desprezamos todos os outros termos da sequência, i.e., todos os termos que não são da forma $\left(b_{0}, b_{1}, \ldots, b_{l+1}, \ldots\right)$ e ainda restam infinitos termos. 
Note que, por construção, as sequências $v^{n_{j}}$ e $w=\left(b_{0}, b_{1}, b_{2}, \ldots\right)$ têm as mesmas $j+1$ primeiras coordenadas, a saber, $b_{0}, b_{1}, \ldots, b_{j}$. Portanto, a distância entre $v^{n_{j}} \mathrm{e}$ $w$ é no máximo $2^{-j}$. Assim, é claro que

$$
\lim _{j \rightarrow \infty} v^{n_{j}}=w
$$

Basicamente o mesmo argumento usado acima funciona para demonstrar o Lema de König em sua formulação mais conhecida, a saber:

Lema 1 (König). Toda árvore infinita em que cada nível é finito possui um caminho infinito, i.e, um galho.

\section{A Versão Infinita Implica a Versão Finita}

Finalmente, vamos usar a Versão Infinita do Teorema de Ramsey e o espaço métrico $\mathcal{K}$, especialmente sua compacidade, para demonstrar a Versão Finita. Vamos, antes, relembrar seu enunciado e compará-lo a um outro muito parecido.

O enunciado explícito da Versão Finita do Teorema de Ramsey é:

Teorema 5 (Ramsey, Versão Finita). Sejam e, $r, k_{1}, k_{2}, \ldots, k_{r} \in \mathbb{N}$, existe $M \in \mathbb{N}$ tal que toda coloração $f:\left(\begin{array}{c}{[M]} \\ e\end{array}\right) \rightarrow C=\left\{c_{1}, c_{2}, c_{3}, \ldots, c_{r}\right\}$ admite, para algum $i \in$ $\{1,2, \ldots, r\}$, um subconjunto $L \subseteq\{1,2, \ldots, M\}$ monocromático de cor $c_{i}$ e tamanho $\geq k_{i}$.

Uma pequena modificação no enunciado, de maneira a deixar sua conclusão mais forte, resulta no teorema abaixo, que, evidentemente, implica o Teorema 5.

A propósito, note que a única diferença entre o teorema 5 e este é a observação final, onde a condição $|L|>\operatorname{Min}(L)$ pode ser intuitivamente interpretada como a exigência de $L$ ser "relativamente grande". Esta nomenclatura será usada na demonstração do teorema, abaixo.

Teorema 6 (Ramsey, Versão de Paris-Harrington). Sejam e, $r, k_{1}, k_{2}, \ldots, k_{r} \in \mathbb{N}$, existe $M \in \mathbb{N}$ tal que toda coloração $f:\left(\begin{array}{c}{[M]} \\ e\end{array}\right) \rightarrow C=\left\{c_{1}, c_{2}, c_{3}, \ldots, c_{r}\right\}$ admite, para algum $i \in\{1,2, \ldots, r\}$, um subconjunto $L \subseteq\{1,2, \ldots, M\}$ monocromático de cor $c_{i}$ e tamanho $\geq k_{i}$. Além disso, $L$ pode ser escolhido de forma que $|L|>\operatorname{Min}(L)$.

Proof. Por absurdo, sejam dados $e, r, k_{1}, k_{2}, \ldots, k_{r} \in \mathbb{N}$ e suponha que, para todo $M \in \mathbb{N}$, exista uma coloração $f^{b}:\left(\begin{array}{c}{[M]} \\ e\end{array}\right) \rightarrow C$ que não admite subconjunto monocromático relativamente grande de cor $c_{i}$ e tamanho $\geq k_{i}$ para nenhum $i \in\{1, \ldots, k\}$. 
Seja $k:=$ Máx $\left\{k_{1}, k_{2}, k_{3}, \ldots, k_{r}\right\}$. Então $f^{b}$ não tem nenhum subconjunto monocromático relativamente grande de tamanho $k$.

Para $M \in \mathbb{N}$, seja $F_{M} \subseteq \mathcal{K}$ tal que $f \in F_{M}$ se, e somente se, $f \Gamma_{\left(\begin{array}{c}{[M]} \\ e\end{array}\right)}$ não admite subconjunto relativamente grande monocromático de tamanho $k$.

Note que $F_{M} \neq \emptyset$. De fato, se $f$ é qualquer extensão de uma $f^{b}$ como acima, temos $f \in F_{M}$.

Note, ainda, que $F_{M}$ é fechado. De fato, tome uma coloração $h:\left(\begin{array}{l}\mathbb{N} \\ e\end{array}\right) \rightarrow C$ no complementar de $F_{M}$. Então $h \uparrow_{\left(\begin{array}{c}{[M]} \\ e\end{array}\right)} \rightarrow C$ possui subconjunto monocromático com $k$ elementos. Esse também será o caso de qualquer outra coloração $\tilde{h}$ cuja distância a $h$ seja $\leq 2^{-M}$, pois as restrições de $h$ e $\tilde{h}$ a $[M]$ coincidem. Ou seja, a bola de centro $h$ e raio $2^{-M}$ está contida no complementar de $F_{M}$. Como a coloração $h$ é arbitrária, o complementar de $F_{M}$ é aberto e $F_{M}$, por sua vez, é fechado.

Como $F_{M} \subseteq \mathcal{K}$ é fechado e $\mathcal{K}$ é compacto, então $F_{M}$ é compacto.

Além disso, claramente

$$
F_{1} \supseteq F_{2} \supseteq F_{3} \supseteq F_{4} \supseteq \ldots
$$

As hipóteses do Teorema dos Compactos Encaixados se verificam, e concluímos que

$$
\bigcap_{i=1}^{\infty} F_{i} \neq \emptyset .
$$

Seja $g \in \bigcap_{i=1}^{\infty} F_{i}$. Note que $g$ contraria a versão infinita do Teorema de Ramsey, por não ter subconjuntos monocromáticos relativamente grandes de tamanho $k$, não tendo, portanto, e com maior razão, subconjuntos infinitos monocromáticos. Temos uma contradição, logo, terminamos.

\section{Algum Contexto Histórico e Comentários Finais}

A discussão sobre os usos do conceito de infinito em matemática é muito ampla e pode, facilmente, ser assunto para muitos outros trabalhos. Gostaríamos, no entanto, de ilustrar, mesmo que de maneira superficial, como toda a discussão apresentada neste texto pode ser inserida naturalmente neste contexto histórico.

A partir dos séculos XIX e XX, pode-se dizer, de forma muito simplificada, que a manipulação formal de conjuntos infinitos como entidades intrínsecas, em particular 
a interpretação de quantificações com escopo em conjuntos infinitos, foi, e de certa forma, tem sido, objeto de uma calorosa controvérsia. Do lado mais simpático à manipulação de infinidades sem grandes restrições, podemos citar Cantor e Hilbert e, do lado diametralmente oposto, defendendo que argumentos matemáticos deveriam ser restritos a raciocínios finitistas de alguma maneira, podemos citar Kroenecker e Brower. Podemos dizer que, para este último grupo, o infinito não se encontrava em lugar nenhum da realidade, sendo apenas uma "ideia abstrata". Não existia na natureza e nem ofereceria base legítima para argumentos racionais precisos. Uma das correntes mais conhecidas deste lado da discussão era a formada pelos Intuicionistas, que não acreditavam que o uso do infinito era aceitável, como ingrediente direto e explícito, em uma demonstração matemática. Os intuicionistas só aceitariam como "entes" matemáticos intrínsecos aqueles que pudessem, em princípio, ser construídos pela mente humana em um número finito de procedimentos. A Aritmética de Peano de Primeira Ordem é um conjunto de axiomas para a aritmética usual dos números inteiros que apenas permite como teoremas aqueles que admitem demonstrações finitistas, aceitas, portanto, mesmo pelo segundo grupo.

Uma pergunta que surge de maneira natural nesse contexto é a seguinte: Não seria conveniente adotar apenas métodos finitistas nas demonstrações matemáticas, tendo em vista que o conteúdo assim produzido não seria objeto das controvérsias acima descritas? Não ousaremos responder a essa pergunta, mas o que vai abaixo ajuda a compreender certos aspectos delicados que deverão ser levados em conta caso alguém deseje formar uma opinião mais substancial sobre o assunto.

Existem pelo menos dois caminhos argumentativos que fornecem demonstrações para o Teorema Finito de Ramsey. Uma análise mais detida da demonstração por induções aninhadas, rapidamente mencionada na primeira seção, mostra que este argumento é finitista e seria, portanto, aceito pelos partidários de ambos os lados da controvérsia acima. Já a segunda demonstração, formulada em linguagem topológica na última seção, seria rejeitada ou, pelo menos, vista, no todo, ou em parte, com desconfiança pelos defensores de algum grau de finitismo, já que os argumentos envolvem manipulação e quantificações diretamente sobre conjuntos infinitos.

Apesar da evidente semelhança nos enunciado dos Teoremas 5 e6, este último não admite demonstrações por meios finitistas. Este é o conteúdo do Teorema abaixo, cuja demonstração utiliza argumentos muito avançados de Lógica Matemática e está fora do escopo deste texto.

Teorema 7 (Paris-Harrington). O Teorema de Ramsey Finito Modificado não é demonstrável na Aritmética de Peano de Primeira Ordem, ou seja, não existe uma 
demonstração para este resultado por métodos exclusivamente finitários.

Os números de Ramsey crescem de maneira muito veloz. De fato, a parte crítica do argumento na demonstração do Teorema de Paris-Harrington consiste em mostrar que a função que leva os parâmetros $\left(e, r, k_{1}, \ldots, k_{r}\right)$ (na hipótese de Ramsey, Versão de Paris-Harrington) no menor $M$ satisfazendo a conclusão cresce de maneira tão violentamente rápida que impossibilita que argumentações finitárias lidem com ela.

A versão de Paris-Harrington do Teorema de Ramsey parece ter sido o primeiro exemplo natural das afirmações indecidíveis em aritmética previstas pelo Teorema da Incompletude de Gödel. Isto é, o enunciado é combinatoriamente natural, não parecendo ter sido explicitamente produzido com o intuito de ser indecidível.

O fato de ser uma afirmação que, apesar de muito natural, não admite demonstrações finitárias torna este resultado algo a ser levado em conta ao refletirmos sobre questões filosóficas envolvendo o uso de argumentos infinitários em Matemática.

\section{References}

[1] MACIEL, J.: Dirichlet e Ramsey: uma bela combinação. São Gonçalo, Rio de Janeiro, FFP-UERJ, 2020.

[2] ALON, N.; SPENCER, J. H.: The probabilistic method. [S.l.]: John Wiley \& Sons, 2004.

[3] STEWART, I.: Almanaque das curiosidades matemáticas: Curiosidades. 1. ed. Rio de Janeiro: Zahar, p. 313, 1945.

[4] BRusamarello, R. L.; Carmelo, E. M.: Paul erdös, o mago. Revista Matemática Universitária, 2018.

[5] CARVALHO, P.C.P.: Combinatória. Ma 12-unidade 11: SBM, 2011.

[6] CONLON, D.: A new upper bound for diagonal ramsey numbers. Annals of Mathematics, 2009.

[7] DOKIC, J.; ENGEL, P.: Frank Ramsey: Truth and Success. [S.l.]: Routledge, 2003.

[8] DORICHEnKO, S.: Um Círculo Matemático de Moscou: Problemas semana-a-semana. 1. ed. Rio de Janeiro: IMPA, p. 247, 2016.

[9] EXOO, G.: A lower bound for $R(5,5)$. Journal of Graph Theory, 1989. 
[10] MCKAY, B. D.; Radziszowski S. P.: Subgraph counting identities and ramsey numbers. Journal of Combinatorial Theory, 1997.

[11] Angeltveit, V.; MCKAY, B. D.: $R(5,5) \leq 48$. ArXiv, 2017.

[12] CARvalho, P. C. P.; MORGado, A. C. O.; FERnAndeZ, P.; PITOMBEIRA, J. B.: Análise Combinatória e Probabilidade. 9a . ed. [S.l.]: SBM, 2006. único.

[13] MORICONI, M.: Qual o problema??: Jogos matemáticos. 1. ed. Rio de Janeiro: Record, p. 112, 2009.

[14] NETO, W. R. S.: O teorema de paris-harrington. PUC-Rio, 2007.

[15] PANSERA, D. R.; VALMÓRBIDA, E.: O princípio da casa dos pombos e suas aplicações. Revista da ORM/SC, n.7, 2010.

[16] PARIS, J.; HARRIS, L.: A mathematical incompleteness in peano arithmetic. Handbook of Mathematical Logic. [S.l.]: North Holland.

[17] SALDANHA, N. C.; TELLES, M.: Spaces of completions of elementary theories and convergence laws for random hypergraphs. Arxiv, 2016. 